\title{
Color Vision
}

National Cancer Institute

\section{Source}

National Cancer Institute. Color Vision. NCI Thesaurus. Code C87177.

The ability to perceive differences in color. 